\title{
Anomalous lateral diffusion in a viscous membrane surrounded by viscoelastic media
}

\author{
S. Komura ${ }^{1}$ (a), S. Ramachandran ${ }^{2}$ and K. SeKi ${ }^{3}$ \\ '1 Department of Chemistry, Graduate School of Science and Engineering, Tokyo Metropolitan University, Tokyo 192- \\ 0397, Japan \\ 2 Physique des Polymères, Université Libre de Bruxelles, Campus Plaine, CP 223, B-1050 Brussels, Belgium \\ 3 National Institute of Advanced Industrial Science and Technology (AIST), Ibaraki 305-8565, Japan
}

PACS 83.60.Bc - Linear viscoelasticity

PACS 87.16.D- - Membranes, bilayers, and vesicles

PACS 87.16.dp - Transport, including channels, pores, and lateral diffusion

\begin{abstract}
We investigate the lateral dynamics in a purely viscous lipid membrane surrounded by viscoelastic media such as polymeric solutions. We first obtain the generalized frequencydependent mobility tensor and focus on the case when the solvent is sandwiched by hard walls. Due to the viscoelasticity of the solvent, the mean square displacement of a disk embedded in the membrane exhibits an anomalous diffusion. An useful relation which connects the mean square displacement and the solvent modulus is provided. We also calculate the cross-correlation of the particle displacements which can be applied for two-particle tracking experiments.
\end{abstract}

Introduction. - Biomembranes are thin twodimensional (2D) fluids which separate inner and outer environments of organelles in cells. The fluidity of biomembranes is guaranteed mainly due to the lipid molecules , which are in the liquid crystalline state at physiological temperatures. Proteins and other molecules embedded in biomembranes undergo lateral diffusion which plays important roles for biological functions 1]. It should be noted, however, that biomembranes are not isolated 2D ' systems, but are coupled to the surrounding polar solvent such as water. Indeed the presence of water is essential for amphiphilic lipid molecules to spontaneously form bilayers by self-assembly.

In the last few decades, it has been recognized that the outer solvent has a significant effect on the membrane dynamics which takes place in 2D. Saffman and Delbrück considered the Brownian motion of a $2 \mathrm{D}$ disk confined in a fluid membrane 2, 3. In their hydrodynamic model, the transfer of membrane momentum to the outer three-dimensional (3D) fluid was taken into account through the boundary conditions at the membrane surfaces. The translational diffusion coefficient in the weak coupling (small disk) limit was shown to exhibit a logarithmic dependence on the disk size. This dependence has

(a) E-mail: komura@tmu.ac.jp been repeatedly tested for various lipid molecules and proteins [4. In the strong coupling (large disk) limit [5], on the other hand, the diffusion coefficient is inversely proportional to the disk size showing the analogy to the 3D Stokes-Einstein relation [6. Such a 3D-like behavior in $2 \mathrm{D}$ membrane is caused by the back flow effect mediated by the bulk solvent.

In this Letter, we discuss the dynamics and responses of membranes when their surrounding solvent is viscoelastic rather than purely viscous. This is a common situation in all eukaryotic cells whose cytoplasm is a soup of proteins and organelles, including a thick sub-membrane layer of actin-meshwork forming a part of the cell cytoskeleton [1. The extra-cellular fluid can also be viscoelastic because it is filled with extracellular matrix or hyaluronic acid gel. In addition to the surrounding solvents, lipid membranes themselves can be viscoelastic [7. Although it turned out that membranes are purely viscous in the latest report [7, their experimental technique using particle tracking microrheology provides us with a new clue to investigate the dynamical responses of lipid bilayers coupled to the surrounding environments under controlled conditions. Recently, viscoelasticity of phospholipid Langmuir monolayers in a liquid-condensed phase was measured using active microrheology [8]. Being motivated by these works, we discuss the mean square displacement (MSD) of a circular 
disk embedded in a 2D sheet by taking into account the viscoelasticity of the surrounding media. This quantity is experimentally measurable in single-particle tracking microrheology [9, 10]. We further calculate the two-particle MSD which is useful for two-point microrheology experiments 11. For both cases, we show that the viscoelasticity of the surrounding media leads to an anomalous diffusion in the $2 \mathrm{D}$ viscous membrane.

Recently, Granek discussed the dynamics of an undulating bilayer membrane surrounded by viscoelastic media 12. He calculated the frequency-dependent transverse (out-of-plane) MSD of a membrane segment and the linear response to external forces. In our theory, we treat the membrane as an infinitely large flat sheet with a $2 \mathrm{D}$ viscosity $\eta$, and consider its lateral dynamics. A similar problem was considered in refs. 13, 14, in which the authors have taken into account the viscoelasticity of the membrane itself because they were originally motivated by the earlier experiment of ref. 77. A more general theory for the dynamics of viscoelastic membranes was given by Levine and MacKintosh 15. In these works, however, the surrounding solvent is assumed to be purely viscous. Similar to Granek's work, the main purpose of our work is to point out the importance of the viscoelasticity of the bulk solvent on the membrane lateral dynamics such as diffusion or linear viscoelastic response. To make this point clear enough, we intentionally treat the membrane as a purely viscous $2 \mathrm{D}$ fluid.

Hydrodynamic model. - We first establish the governing hydrodynamic equations for our model. As shown in fig. 1. the fluid membrane, fixed in the $x y$-plane at $z=0$, is embedded in a bulk solvent that is further bounded by hard walls at $z= \pm h$. Let $\mathbf{v}(\mathbf{r}, t)$ be the $2 \mathrm{D}$ velocity of the membrane fluid at position $\mathbf{r}=(x, y)$ and at time $t$. We assume that the membrane is incompressible;

$$
\nabla \cdot \mathbf{v}=0 .
$$

We also work in the low-Reynolds number regime so that the inertial effects can be neglected. Then the Stokes equation for the fluid membrane is given by

$$
\rho \frac{\partial \mathbf{v}}{\partial t}=\eta \nabla^{2} \mathbf{v}-\nabla p+\mathbf{f}_{\mathbf{s}}+\mathbf{F},
$$

where $\rho$ is the membrane 2D density, $\eta$ the membrane $2 \mathrm{D}$ constant viscosity, $p$ the in-plane pressure, $\mathbf{f}_{\mathrm{s}}$ the force due to the solvent described later, and $\mathbf{F}$ is any other force acting on the membrane. Notice that $\nabla$ stands for the $2 \mathrm{D}$ differential operator.

Next we give the equations for the surrounding viscoelastic solvent. The upper $(z>0)$ and the lower $(z<0)$ regions of the solvent are denoted by "+" and "-", respectively. The velocities and pressures in these regions are written as $\mathbf{v}^{ \pm}(\mathbf{r}, z, t)$ and $p^{ \pm}(\mathbf{r}, z, t)$, respectively. Similar to the fluid membrane, the solvent is also assumed to be incompressible;

$$
\tilde{\nabla} \cdot \mathbf{v}^{ \pm}=0 .
$$

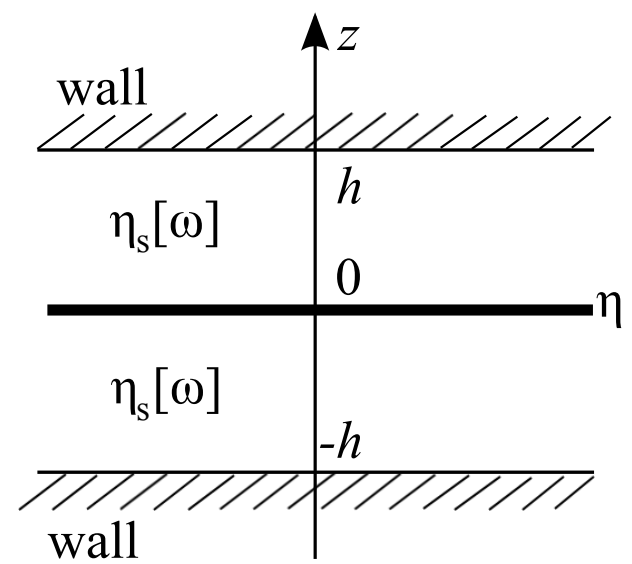

Fig. 1: Schematic picture showing a planar viscous membrane at $z=0$ with $2 \mathrm{D}$ constant viscosity $\eta$. It is sandwiched by a viscoelastic solvent of $3 \mathrm{D}$ frequency-dependent viscosity $\eta_{\mathrm{s}}[\omega]$. Two impenetrable walls are located at $z= \pm h$ bounding the solvent.

The Stokes equation for the viscoelastic solvent is written as

$$
\rho_{\mathrm{s}} \frac{\partial \mathbf{v}^{ \pm}}{\partial t}=\tilde{\nabla} \cdot \boldsymbol{\sigma}^{ \pm}-\tilde{\nabla} p^{ \pm}
$$

where $\rho_{\mathrm{s}}$ is the solvent 3D density (assumed to be the same for both solvents), and $\tilde{\nabla}$ indicates the 3D differential operator. Within the linear viscoelasticity approximation, the stress tensor $\boldsymbol{\sigma}^{ \pm}$in the above equation is given by the following constitutive relation [16]

$$
\boldsymbol{\sigma}^{ \pm}(t)=2 \int_{-\infty}^{t} \mathrm{~d} t^{\prime} \eta_{\mathrm{s}}\left(t-t^{\prime}\right) \mathbf{D}^{ \pm}\left(t^{\prime}\right)
$$

where $\eta_{\mathbf{s}}(t)$ is the time-dependent solvent viscosity (assumed to be the same for both solvents). The rate-ofstrain tensor is given by $\mathbf{D}^{ \pm}=\left[\tilde{\nabla} \mathbf{v}^{ \pm}+\left(\tilde{\nabla} \mathbf{v}^{ \pm}\right)^{\mathrm{T}}\right] / 2$, where the superscript " $\mathrm{T}$ " denotes the transpose. The surrounding viscoelastic solvent exerts force $\mathbf{f}_{\mathrm{s}}$ on the membrane as considered in eq. (2). It is given by the projection of $\left(\boldsymbol{\sigma}^{+}-\boldsymbol{\sigma}^{-}\right)_{z=0} \cdot \hat{\mathbf{e}}_{z}$ on the $x y$-plane, where $\hat{\mathbf{e}}_{z}$ is the unit vector along the $z$-axis. We employ the stick boundary conditions at any time, i.e., the equalities of the velocities, at $z=0$ and $z= \pm h$. When the solvent is purely viscous, this model reduces to that considered by the present authors [17.

It is convenient to perform the 2D Fourier transform in space and the Fourier-Laplace (or one-sided Fourier) transform in time for any function $f(\mathbf{r}, t)$ as defined by $f[\mathbf{k}, \omega]=\int_{-\infty}^{\infty} \mathrm{d}^{2} r \int_{0}^{\infty} \mathrm{d} t f(\mathbf{r}, t) \exp [-i(\mathbf{k} \cdot \mathbf{r}+\omega t)]$, where $\mathbf{k}=\left(k_{x}, k_{y}\right)$ is the $2 \mathrm{D}$ wavevector and $\omega$ the angular frequency. Assuming that fluids are at rest at $t=0$, we calculate $\mathbf{f}_{\mathrm{s}}$ and obtain the membrane velocity as $\mathbf{v}[\mathbf{k}, \omega]=$ $\mathbf{G}[\mathbf{k}, \omega] \cdot \mathbf{F}[\mathbf{k}, \omega]$, where $\mathbf{G}[\mathbf{k}, \omega]$ is the frequency-dependent mobility tensor. Following the similar procedure described 
in refs. [13, 14, we obtain

$$
G_{\alpha \beta}[\mathbf{k}, \omega]=\frac{\delta_{\alpha \beta}-k_{\alpha} k_{\beta} / k^{2}}{\eta k^{2}+2 \eta_{\mathbf{s}}[\omega] k^{\prime} \operatorname{coth}\left(k^{\prime} h\right)+i \omega \rho},
$$

where $k^{\prime}=k\left(1+i \omega \rho_{\mathrm{s}} / \eta_{\mathrm{s}}[\omega] k^{2}\right)^{1 / 2}, k=|\mathbf{k}|$ and $\alpha, \beta=$ $x, y$. In the limit of $h \rightarrow \infty$, which we call as the "free membrane case", the above mobility tensor reduces to

$$
G_{\alpha \beta}^{\mathrm{free}}[\mathbf{k}, \omega]=\frac{\delta_{\alpha \beta}-k_{\alpha} k_{\beta} / k^{2}}{\eta k^{2}+2 \eta_{\mathrm{s}}[\omega] k \sqrt{1+i \omega \rho_{\mathrm{s}} / \eta_{\mathrm{s}}[\omega] k^{2}}+i \omega \rho},
$$

which was given in ref. [13. The opposite limit of $h \rightarrow 0$ is called as the "confined membrane case" and the corresponding mobility tensor becomes

$$
G_{\alpha \beta}^{\mathrm{con}}[\mathbf{k}, \omega]=\frac{\delta_{\alpha \beta}-k_{\alpha} k_{\beta} / k^{2}}{\eta\left(k^{2}+\kappa^{2}[\omega]\right)},
$$

where we have introduced $\kappa[\omega]$ whose square is defined as

$$
\kappa^{2}[\omega]=\frac{2 \eta_{\mathrm{s}}[\omega]}{\eta h}+\frac{i \omega \rho}{\eta} .
$$

In the limit of $\omega \rightarrow 0$, eq. (6) reduces to the static mobility tensor obtained in ref. [17]. The free membrane case was originally considered by Saffman and Delbrück [2, 3, and the relevant length scale is the Saffman-Delbrück length $\eta / \eta_{\mathrm{s}}$ beyond which the membrane feels the presence of the outer solvent. On the other hand, the confined membrane case corresponds to that of a supported membrane close to the substrate as considered by Evans and Sackmann [18] and later generalized by us [19. Here the corresponding hydrodynamic screening length is set by the geometric mean of the Saffman-Delbrück length $\eta / \eta_{\mathrm{s}}$ and the distance $h$ between the membrane and the wall, i.e., $\sqrt{\eta h / \eta_{\mathrm{s}}}[20$. Notice that the presence of the second wall only doubles the screening effect. In the following, we shall mainly consider the confined membrane case which allows us to treat most of the calculations analytically. This is mainly because $\kappa[\omega]$ does not depend on $k$. However, it should be noted that the limiting expression of eq. (8) gives a reasonable approximation to the full expression of eq. (6) even for $h \sim \eta / \eta_{\mathrm{s}}$ at least for a large enough moving object 20,21.

Concerning the viscoelasticity of the surrounding solvent, we assume that its complex modulus obeys the power-law behavior such that $G_{\mathrm{s}}[\omega]=G_{0}(i \omega)^{\alpha}$ with $\alpha<1$, as generally argued by Granek [12. This behavior is commonly observed for various polymeric solutions at high frequencies. Examples are $\alpha=1 / 2$ and $\alpha=2 / 3$ for Rouse and Zimm dynamics, respectively [22], $\alpha=3 / 4$ for semi-dilute solutions of semi-flexible polymers such as actin filaments [23]. From the viewpoint of particle-tracking microrheology experiment 24, 25, it is more convenient to work in the Laplace domain defined by $\tilde{f}(s)=\int_{0}^{\infty} \mathrm{d} t f(t) e^{-s t}$. Since we have been working in the Fourier-Laplace domain, it is straightforward to convert to the Laplace domain by substituting $s=i \omega$. From
$\tilde{G}_{\mathrm{s}}(s)=G_{0} s^{\alpha}$, the Laplace transform of the solvent viscosity behaves as $\tilde{\eta}_{\mathrm{s}}(s)=\tilde{G}_{\mathrm{s}}(s) / s=G_{0} s^{\alpha-1}$. Then eq. (9) simply becomes

$$
\tilde{\kappa}^{2}(s)=\frac{2 G_{0} s^{\alpha-1}}{\eta h}+\frac{\rho s}{\eta} \approx \frac{2 G_{0} s^{\alpha-1}}{\eta h},
$$

where we have dropped the inertial term at the end. This approximation is justified when $G_{0} s^{\alpha-2} / \rho h \gg 1$, which is always valid for $s \rightarrow 0$ corresponding to the long-time behavior.

Single-particle tracking. - For the description of the Brownian motion of a circular disk confined in a membrane, we basically follow the formulation in ref. 26. Let $a$ and $m$ be the radius and the mass of the disk, respectively. The effective generalized Langevin equation is written as 27 .

$$
m^{*} \frac{\mathrm{d}}{\mathrm{d} t} U(t)=-\int_{-\infty}^{t} \mathrm{~d} t^{\prime} \lambda\left(t-t^{\prime}\right) U\left(t^{\prime}\right)+R(t),
$$

where $U(t)$ is the velocity of the disk, and $\lambda(t)$ is the timedependent drag coefficient given below. The random force $R(t)$ satisfies the fluctuation dissipation theorem (FDT) when averaged over the ensemble of molecular motions; i.e., $\langle R(t)\rangle=0$ and $\left\langle R\left(t_{0}\right) R\left(t_{0}+t\right)\right\rangle=k_{\mathrm{B}} T \lambda(t)$, where $k_{\mathrm{B}}$ is the Boltzmann constant and $T$ the temperature. In ref. [26, it was shown that the renormalized mass is given by $m^{*}=m+\pi \rho a^{2}$ which takes into account the additional inertia due to the drag from the fluid membrane. Furthermore, the Laplace transform of $\lambda(t)$ is calculated to be

$$
\tilde{\lambda}(s)=\frac{2 \pi \tilde{\eta}_{\mathrm{s}}(s) a^{2}}{h}+4 \pi \eta \frac{\tilde{\kappa}(s) a K_{1}[\tilde{\kappa}(s) a]}{K_{0}[\tilde{\kappa}(s) a]},
$$

where $K_{0}[x]$ and $K_{1}[x]$ are modified Bessel functions of the second kind, order zero and one, respectively.

Following the standard procedure to obtain the MSD [27, one can relate its Laplace transform and $\tilde{\lambda}(s)$ through

$$
\left\langle\Delta \tilde{\mathbf{r}}^{2}(s)\right\rangle=\frac{4 k_{\mathrm{B}} T}{s^{2} \tilde{\lambda}(s)},
$$

where the number of degrees of freedom tracked in the MSD is chosen to be two. For general $t$, application of the inverse Laplace transform provides us with the timedependent MSD;

$$
\left\langle\Delta \mathbf{r}^{2}(t)\right\rangle=\frac{1}{2 \pi i} \int_{c-i \infty}^{c+i \infty} \mathrm{d} s \frac{4 k_{\mathrm{B}} T}{s^{2} \tilde{\lambda}(s)} e^{s t} .
$$

In order to demonstrate how to use the above relations, we consider here the limit of a large disk size $a \rightarrow \infty$ $(\tilde{\kappa}(s) a \gg 1)$ so that the drag coefficient in eq. (12) is dominated by the first term, i.e., $\tilde{\lambda}(s) \approx 2 \pi \tilde{\eta}_{\mathrm{s}}(s) a^{2} / h$. Then the Laplace transformed MSD in eq. (13) becomes

$$
\left\langle\Delta \tilde{\mathbf{r}}^{2}(s)\right\rangle=\frac{2 k_{\mathrm{B}} T h}{\pi a^{2} s^{2} \tilde{\eta}_{\mathrm{s}}(s)}=\frac{2 k_{\mathrm{B}} T h}{\pi a^{2} s \tilde{G}_{\mathrm{s}}(s)} .
$$


This is the equation which relates the observed 2D MSD to the modulus of the surrounding bulk solvent (rather than the membrane). In other words, we can extract the solvent $3 \mathrm{D}$ information by using the $2 \mathrm{D}$ information due to the motion of a disk in the membrane. Once $\tilde{G}_{\mathrm{s}}(s)$ is obtained from the experiment, the frequency dependence of the storage and the loss moduli can be deduced by identifying $G_{\mathrm{s}}[\omega]=G_{\mathrm{s}}^{\prime}[\omega]+i G_{\mathrm{s}}^{\prime \prime}[\omega]=\tilde{G}_{\mathrm{s}}(s=i \omega)$. Notice that these two representations are equivalent because $G_{\mathrm{s}}^{\prime}[\omega]$ and $G_{\mathrm{s}}^{\prime \prime}[\omega]$ are related by the Kramers-Kronig relation 24, 25, 27.

On the other hand, suppose the modulus of the bulk solvent is known a priori to behave as $\tilde{G}_{\mathrm{S}}(s)=G_{0} s^{\alpha}$ from independent experiments, the MSD can be simply obtained from eqs. (14) and (15) as

$$
\left\langle\Delta \mathbf{r}^{2}(t)\right\rangle=\frac{2 k_{\mathrm{B}} T h}{\pi a^{2} G_{0} \Gamma[1+\alpha]} t^{\alpha},
$$

where $\Gamma[x]$ is the gamma function. This calculation shows that the viscoelasticity of the solvent results in a subdiffusive time dependence of the MSD. Since $\alpha<1$, the viscoelasticity slows down the normal diffusion process. This is the main result of this Letter. Compared with the $3 \mathrm{D}$ case 9,10 , the above expression is unique because it is proportional to $h / a^{2}$. This $1 / a^{2}$-dependence arises from the mass conservation in $2 \mathrm{D}$ rather than the momentum conservation [17.

In the limit of a small disk size $a \rightarrow 0(\tilde{\kappa}(s) a \ll 1)$, the situation is more complicated. In this case, the drag coefficient asymptotically behaves as

$$
\tilde{\lambda}(s) \approx 4 \pi \eta\left[\ln \left(\frac{2}{\tilde{\kappa}(s) a}\right)-\gamma\right]^{-1},
$$

where $\gamma=0.5772 \cdots$ is Euler's constant. By using eq. (10) for $\tilde{\kappa}(s)$, a similar calculation yields

$$
\left\langle\Delta \mathbf{r}^{2}(t)\right\rangle \approx \frac{k_{\mathrm{B}} T}{2 \pi \eta} t\left[\ln \left(\frac{2 \eta h t^{\alpha-1}}{G_{0} a^{2}}\right)+(\alpha-3) \gamma-\alpha+1\right] .
$$

Since $\alpha<1$, this MSD grows like $t \ln (1 / t)$. Such a logarithmic correction leads to a time-dependent diffusivity. It should be noted, however, that this asymptotic expression is valid only when $G_{0} a^{2} t^{1-\alpha} / \eta h \ll 1$. We also mention that all the above expressions recover our previous results when the solvent is purely viscous, i.e., $\alpha \rightarrow 1$ [19].

In fig. 2. we plot the result of numerical inverse Laplace transform of eq. (14) with the full drag coefficient eq. (12) when $\alpha=1 / 2$, and compared it with the asymptotic expressions; eqs. (16) and (18). Here the dimensionless MSD and time are given by $\left(2 \pi \eta / k_{\mathrm{B}} T\right)\left(\eta h / 2 G_{0} a^{2}\right)^{1 /(\alpha-1)}\left\langle\Delta \mathbf{r}^{2}(t)\right\rangle$ and $\bar{t}=$ $\left(\eta h / 2 G_{0} a^{2}\right)^{1 /(\alpha-1)} t$, respectively. As mentioned above, eq. (18) is valid only for short time $(\bar{t} \ll 1)$, while eq. (16) describes the asymptotic long-time behavior $(\bar{t} \gg$ $\left.16^{1 /(1-\alpha)}\right)$. The intermediate time region is described by an apparent power-law $\bar{t}^{(1+\alpha) / 2}$, which will be explained elsewhere.

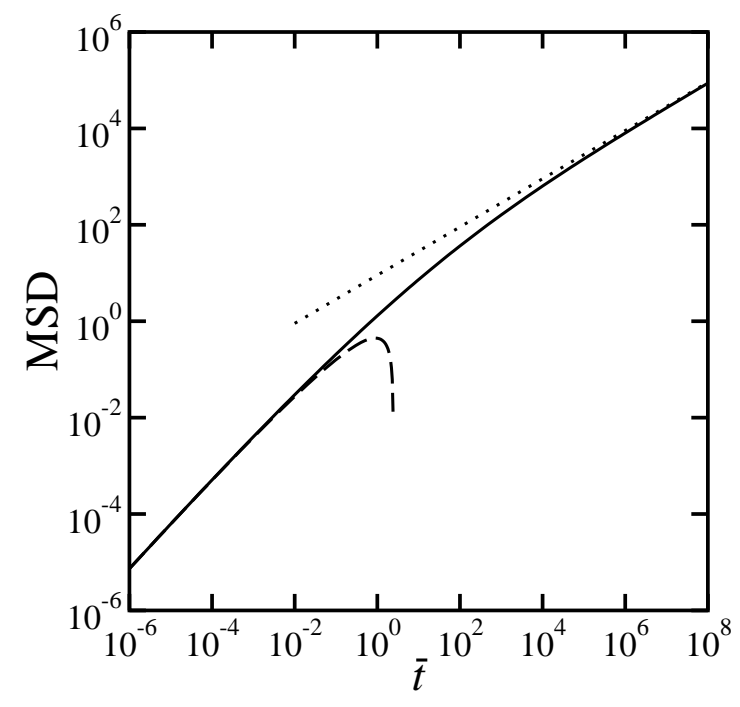

Fig. 2: Scaled MSD as a function of scaled time $\bar{t}$ when $\alpha=1 / 2$. These dimensionless quantities are defined in the text. The dotted and dashed lines correspond to the asymptotic expressions given by eqs. (16) and (18), respectively.

Two-particle tracking. - So far, we have discussed the motion of a single disk of finite radius $a$. As discussed in ref. [25, there are several advantages to perform multiparticle microrheology. For example, long-time convective drift can be automatically subtracted in this method so that measurements of probe self-diffusivities become possible over longer times. Multi-particle techniques can be also used to investigate heterogeneous materials. Here we discuss the effects of the solvent viscosity on the crosscorrelation of two distinct probe positions, namely, twopoint microrheology [11. We show below that the distance between the two points essentially corresponds to the size of a disk in single-particle microrheology.

Consider a pair of point particles embedded in the membrane undergoing Brownian motion separated by a $2 \mathrm{D}$ vector $\mathbf{r}$. The quantity of interest is the cross-correlation of the particle displacements $\left\langle\Delta r_{\alpha}^{1}(0) \Delta r_{\beta}^{2}(t)\right\rangle_{r}$, where $\Delta r_{\alpha}^{i}$ is the displacement of the particle $i(=1,2)$ along the axis $\alpha$ $(=x, y)$. We also define the $x$-axis to be along the line connecting the two particles, i.e., $\mathbf{r}=r \hat{\mathbf{e}}_{x}$. According to the FDT, this correlation function is related to the coupling mobility $M_{\alpha \beta}(t)$ in the Laplace domain as 25

$$
\left\langle\Delta \tilde{r}_{\alpha}^{1} \Delta \tilde{r}_{\beta}^{2}(s)\right\rangle_{r}=\frac{2 k_{\mathrm{B}} T}{s^{2}} \tilde{M}_{\alpha \beta}(r, s),
$$

for sufficiently large $r$. The above equation is the analog of eq. (13) for the two-particle tracking. Since $M_{x y}=0$ by symmetry, we consider the longitudinal coupling mobility $M_{\mathrm{L}}=M_{x x}$ and the transverse one $M_{\mathrm{T}}=M_{y y}$. Note that the coupling mobility is not the inverse of the coupling resistance in the multi-particle case 25. By utilizing the results in refs. 17, 28, 29, one can obtain these mobilities analytically. 
First the Laplace transform of the longitudinal coupling mobility turns out to be

$$
\tilde{M}_{\mathrm{L}}(r, s)=\frac{1}{2 \pi \eta}\left[\frac{1}{(\tilde{\kappa}(s) r)^{2}}-\frac{K_{1}[\tilde{\kappa}(s) r]}{\tilde{\kappa}(s) r}\right] .
$$

In the limit of a large distance $r \rightarrow \infty(\widetilde{\kappa}(s) r \gg 1)$, the above expression can be approximated as

$$
\tilde{M}_{\mathrm{L}}(r, s) \approx \frac{1}{2 \pi \eta} \frac{1}{(\tilde{\kappa}(s) r)^{2}} .
$$

As in the calculation of MSD of a single disk, we use eq. (10) for $\tilde{\kappa}(s)$ and perform the inverse Laplace transform of eq. (19). Then we obtain

$$
\left\langle\Delta r_{x}^{1}(0) \Delta r_{x}^{2}(t)\right\rangle_{r} \approx \frac{k_{\mathrm{B}} T h}{2 \pi G_{0} \Gamma[1+\alpha]} \frac{t^{\alpha}}{r^{2}}
$$

The subdiffusive dependence on time and the $1 / r^{2}$ dependence on distance is analogous to eq. (16), implying that $r$ corresponds to $a$.

In the limit of a small distance $r \rightarrow 0(\tilde{\kappa}(s) r \ll 1)$, on the other hand, eq. (20) asymptotically behaves as

$$
\tilde{M}_{\mathrm{L}}(r, s) \approx \frac{1}{4 \pi \eta}\left[\ln \left(\frac{2}{\tilde{\kappa}(s) r}\right)-\gamma+\frac{1}{2}\right] .
$$

Following the same process as above, the cross-correlation function asymptotically behaves as

$$
\begin{aligned}
\left\langle\Delta r_{x}^{1}(0) \Delta r_{x}^{2}(t)\right\rangle_{r} & \approx \frac{k_{\mathrm{B}} T}{4 \pi \eta} t\left[\ln \left(\frac{2 \eta h t^{\alpha-1}}{G_{0} r^{2}}\right)\right. \\
& +(\alpha-3) \gamma-\alpha+2]
\end{aligned}
$$

which is valid for $G_{0} r^{2} t^{1-\alpha} / \eta h \ll 1$. Notice that eq. (24) is also analogous to eq. (18).

Next the transverse coupling mobility is given by

$$
\tilde{M}_{\mathrm{T}}(r, s)=\frac{1}{2 \pi \eta}\left[K_{0}[\tilde{\kappa}(s) r]+\frac{K_{1}[\tilde{\kappa}(s) r]}{\tilde{\kappa}(s) r}-\frac{1}{(\tilde{\kappa}(s) r)^{2}}\right] \text {. }
$$

The large distance limit $(r \rightarrow \infty)$ and the small distance limit $(r \rightarrow 0)$ of this expression are

$$
\tilde{M}_{\mathrm{T}}(r, s) \approx-\frac{1}{2 \pi \eta} \frac{1}{(\tilde{\kappa}(s) r)^{2}},
$$

and

$$
\tilde{M}_{\mathrm{T}}(r, s) \approx \frac{1}{4 \pi \eta}\left[\ln \left(\frac{2}{\tilde{\kappa}(s) r}\right)-\gamma-\frac{1}{2}\right],
$$

respectively. Since these forms differ from eqs. (21) and (23) only by a sign, we do not repeat here the same calculations. However, as far as the time dependence of $\left\langle\Delta r_{y}^{1}(0) \Delta r_{y}^{2}(t)\right\rangle_{r}$ is concerned, it is essentially given by eqs. (22) and (24).
Discussion. - In this Letter, we have discussed the dynamics in a purely viscous lipid membrane surrounded by viscoelastic solvents such as polymeric solutions. Using the generalized frequency-dependent mobility tensor for the confined membrane case, we calculated the MSD of a disk embedded in the membrane and obtained some asymptotic expressions. The obtained MSD exhibits an anomalous diffusion reflecting the viscoelastic property of the bulk solvent. For single-particle microrheology experiments, we presented an useful relation which connects the MSD and the solvent modulus in the Laplace domain when the size of the disk is large enough. We also obtained the cross-correlation of the particle displacements which can be used for two-particle tracking experiments. Our theory can be applied not only for lipid membranes but also for Langmuir monolayers.

It is worthwhile to point out the implicit assumptions which are used in the present theory 25. First we have assumed that the system obeys the FDT which allows us to relate the thermal fluctuations of the probe disk directly to the time-dependent drag coefficient (see eq. (13)) or the coupling mobility (see eq. (19)). However, in non-equilibrium situations with membrane transport proteins, for instance, the FDT can be violated and the deviation from the present theory may arise. Recently, it was reported that an in vitro model system consisting of a cross-linked actin network with embedded forcegenerating myosin motors strongly violates FDT [30]. The second crucial assumption is that the generalized drag coefficient or the coupling mobility are given precisely by their Newtonian analogs, but with the Newtonian viscosity $\eta_{\mathrm{s}}$ replaced by the frequency-dependent complex viscosity $\eta_{\mathrm{s}}[\omega]$ at all frequencies (see eq. (5)). This is not an obvious assumption, but it has proved to be quite successful for any probe motion in a simple linear viscoelastic material under various conditions at least in 3D [25].

Some caution is required when applying our theory to experiments if the viscoelastic solvent is, for instance, a semi-dilute polymer solution. For single-particle tracking, our continuum approach is valid for inclusion sizes much larger than the mesh size of the network. This can be relevant such as for a micron-size membrane domain and a network correlation length of tens of nanometers. However our theory may not be applicable for small membrane proteins which feel the solvent as purely viscous. For twoparticle tracking, the distance between the two point particles (which can be small membrane proteins) should be larger than the network mesh size. In order to obtain the full time behavior of the particle motion, one should use a viscoelastic modulus that is dependent both on wavevector and frequency. On the other hand, any simple fluid can be effectively viewed as viscoelastic at very high molecular frequencies 31]. Our model can be also used to study such a high frequency dynamics by using a Maxwell model for the solvent.

In the present work, we have mainly discussed the confined membrane case in order to obtain analytical expres- 
sions. Unfortunately, a single analytic expression of the drag coefficient for the whole range of the disk size is not known for the free membrane case. However, in the limit of a large disk size $a \rightarrow \infty$, the asymptotic expression was obtained by Hughes et al. as $\lambda_{\text {free }} \approx 16 \eta_{\mathrm{s}} a$ which depends only on the solvent viscosity and is proportional to the size [5]. Assuming the replacement of the solvent viscosity with the time-dependent one as discussed above, the MSD of the disk becomes

$$
\left\langle\Delta \tilde{\mathbf{r}}^{2}(s)\right\rangle_{\text {free }}=\frac{k_{\mathrm{B}} T}{4 a s^{2} \tilde{\eta}_{\mathrm{s}}(s)}=\frac{k_{\mathrm{B}} T}{4 a s \tilde{G}_{\mathrm{s}}(s)} .
$$

When the modulus of the bulk solvent behaves as $\tilde{G}_{\mathrm{s}}(s)=$ $G_{0} s^{\alpha}$ as before, the MSD in the free membrane case is given by

$$
\left\langle\Delta \mathbf{r}^{2}(t)\right\rangle_{\text {free }}=\frac{k_{\mathrm{B}} T}{4 a G_{0} \Gamma[1+\alpha]} t^{\alpha},
$$

which is again a subdiffusive behavior. In the opposite limit of $a \rightarrow 0$, the Laplace transformed drag coefficient can be written as 2,3 ,

$$
\tilde{\lambda}_{\text {free }}(s) \approx 4 \pi \eta\left[\ln \left(\frac{\eta}{\tilde{\eta}_{\mathrm{s}}(s) a}\right)-\gamma\right]^{-1} .
$$

It then follows that

$$
\left\langle\Delta \mathbf{r}^{2}(t)\right\rangle_{\text {free }}=\frac{k_{\mathrm{B}} T}{\pi \eta} t\left[\ln \left(\frac{\eta t^{\alpha-1}}{G_{0} a}\right)+(\alpha-2) \gamma-\alpha+1\right],
$$

for $G_{0} a t^{1-\alpha} / \eta \ll 1$. Although the above argument is not rigorous, it essentially captures the anomalous diffusion behavior in the free membrane case. For the twoparticle tracking case, however, the corresponding crosscorrelation function can be obtained without any ambiguity since both the longitudinal and transverse coupling mobilities were analytically obtained for the free membrane case 29.

Currently we are extending our model to the case when the two solvents have asymmetric viscoelastic properties or when the disk is actively driven. Additional effects such as the finite curvature of vesicles or the out-of-plane deformation of the membrane should also be taken into account.

\section{$* * *$}

We thank C.-Y. D. Lu for useful discussions. This work was supported by Grant-in-Aid for Scientific Research (grant No. 21540420) from the MEXT of Japan. SK also acknowledges the supported by the JSPS Coreto-Core Program "International research network for nonequilibrium dynamics of soft matter".

\section{REFERENCES}

[1] Alberts B., Johnson A., Lewis J., Raff M., Roberts K. and WAlter P., Molecular Biology of the Cell (Garland Science) 2008.
[2] Saffman P. G. and Delbrück M., Proc. Natl. Acad. Sci. U.S.A., 72 (1975) 3111.

[3] Saffman P. G., J. Fluid Mech., 73 (1976) 593.

[4] Gambin Y., Lopez-Esparza R., Reffay M., Sierecki E., Gov N. S., Genest M., Hodges R. S. and Urbach W., Proc. Natl. Acad. Sci. U.S.A., 103 (2006) 2098.

[5] Hughes B. D., Pailthorpe B. A. and White L. R., J. Fluid Mech., 110 (1981) 349.

[6] Landau L. D. and Lifshitz E. M., Fluid Mechanics (Pergamon Press) 1987.

[7] Harland C. W., Bradley M. J. and Parthasarathy R., Proc. Natl. Acad. Sci. U.S.A., 107 (2010) 19146; Proc. Natl. Acad. Sci. U.S.A., 108 (2011) 14705.

[8] Choi S. Q., Steltenkamp S., Zasadzinski J. A. and Squires T. M., Nature Comm., 2 (2011) 312.

[9] Mason T. G. and Weitz D. A., Phys. Rev. Lett., 74 (1995) 1250.

[10] Mason T. G., Ganesan K., van Zanten J. H., Wirtz D. and Kuo S. C., Phys. Rev. Lett., 79 (1997) 3282.

[11] Crocker J. C., Valentine M. T., Weeks E. R., Gisler T., Kaplan P. D., Yodh A. G. and Weitz D. A., Phys. Rev. Lett., 85 (2000) 8888.

[12] Granek R., Soft Matter, 7 (2011) 5281.

[13] Camley B. A. and Brwon F. L. H., Phys. Rev. E, 84 (2011) 021904.

[14] Han T. and HaAtaja M., Phys. Rev. E, 84 (2011) 051903.

[15] Levine A. J. and MacKintosh F. C., Phys. Rev. E, 66 (2002) 061606.

[16] Bird R. B. and Hassager O., Dynamics of Polymeric Liquids, Vol.1 (Wiley Interscience) 1987.

[17] Ramachandran S., Komura S., Seki K. and Gompper G., Eur. Phys. J. E, 34 (2011) 46.

[18] Evans E. and Sackmann E., J. Fluid Mech., 194 (1988) 553.

[19] Seki K., Ramachandran S. and Komura S., Phys. Rev. E, 84 (2011) 021905.

[20] Stone H. and Ajdari A., J. Fluid Mech., 369 (1998) 151.

[21] Ramachandran S., Komura S., Seki K. and Imai M., Soft Matter, 7 (2011) 1524.

[22] Doi M. and Edwards S. F., The Theory of Polymer Dynamics (Oxford University Press) 1986.

[23] Gittes F., Schnurr B., Olmsted P. D., MacKintosh F. C. and Schmidt C. F., Phys. Rev. Lett., 79 (1997) 3286.

[24] Mason T. G., Rheol. Acta, 39 (2000) 371.

[25] Squires T. M. and Mason T. G., Annu. Rev. Fluid Mech., 42 (2010) 413.

[26] Seki K. and Komura S., Phys. Rev. E, 47 (1993) 2377.

[27] Kubo R., Toda M. and Hashitsume N., Statistical Physics II (Springer-Verlag) 1991.

[28] Ramachandran S., Komura S. and Gompper G., EPL, 89 (2010) 56001.

[29] Oppenheimer N. and Diamant H., Phys. Rev. E, 82 (2010) 041912.

[30] Mizuno D., Tardin C., Schmidt C. F. and MacKinTOsh F. C., Science, 315 (2007) 370.

[31] Zwanzig R. and Bixon M., Phys. Rev. A, 2 (1970) 2005. 